\title{
Article \\ Performance evaluation for repair of HSGc-C5 carcinoma cell using Geant4-DNA
}

\author{
Dousatsu Sakata ${ }^{1,+*(D)}$, Masao Suzuki ${ }^{2,+}$, Ryoichi Hirayama ${ }^{2,+}$, Yasushi Abe ${ }^{1}$, Masayuki Muramatsu ${ }^{1}$, Shinji Sato \\ ${ }^{1}$, Oleg Belov ${ }^{3,4}$, Ioanna Kyriakou ${ }^{5}$, Dimitris Emfietzoglou ${ }^{5}$, Susanna Guatelli ${ }^{6}$, Sebastien Incerti ${ }^{7}$ and Taku \\ Inaniwa 1
}

1 Department of Accelerator and Medical Physics, Institute for Quantum Medical Science, QST, Chiba, Japan

2 Department of Charged Particle Therapy Research, Institute for Quantum Medical Science, QST, Chiba, Japan

3 Joint Institute for Nuclear Research, Dubna, Russia

4 Dubna State University, Dubna, Russia

5 Medical Physics Laboratory, University of Ioannina, Medical School, GR-45110 Ioannina, Greece

6 Centre For Medical Radiation Physics, University of Wollongong, Wollongong, Australia

7 Université de Bordeaux, CNRS/IN2P3, UMR5797, Centre d'Études Nucléaires de Bordeaux Gradignan, 33175 Gradignan, France

* Correspondence: sakata.dousatsu@qst.go.jp

+ These authors contributed equally to this work.

Simple Summary: To evaluate the repair performance of HSGc-C5 carcinoma cell against radiation induced DNA damage, a Geant4-DNA application for radiobiological research was extended by using newly measured experimental data acquired in this study. Concerning fast- and slowDNA rejoining, the two-lesion kinetics (TLK) model parameters were adequately optimized (the repair speeds of each process were reasonably close to the DNA-rejoining speed of the NHEJ and HR processes). The lethality rates of the DNA damage induced by complex DSBs and binaryrepair were approximately $3 \%$ and $45 \%$, respectively. Using the optimized repair parameters, the Geant4-DNA simulation was able to predict the SF and the DNA-repair kinetics.

\begin{abstract}
Track-structure Monte Carlo simulations are useful tools to evaluate initial DNA damage induced by irradiation. In the previous study, we have developed a Gean4-DNA-based application to estimate the cell surviving fraction of V79 cells after irradiation, bridging the gap between the initial DNA damage and the DNA-rejoining kinetics by means of the two-lesion kinetics (TLK) model. However, since the DNA repair performance depends on cell line, the same model parameters cannot be used for the different cell lines. Thus, we extended the Geant4-DNA application with an updated TLK model for the evaluation of DNA damage repair performance in HSGc-C5 carcinoma cells which are typically used for evaluating proton/carbon radiation treatment effects. For this evaluation, we also performed experimental measurements for cell surviving fractions and DNA-rejoining kinetics of the HSGc-C5s cells. Concerning fast- and slow-DNA rejoining, the TLK model parameters were adequately optimized with the simulated initial DNA damage. Using the optimized TLK model, the Geant4-DNA simulation is now able to predict cell survival and DNA-rejoining kinetics for HSGc-C5s cells.
\end{abstract}

Keywords: Geant4-DNA; DNA repair; Cell surviving fraction

\section{Introduction}

Radiation treatment is one of the most widely used therapeutic techniques for cancer treatment aimed at depriving tumor cells of their reproductive potential [1]. The investigation of biological responses to radiation, such as reproductive cell death, have emerged as a multi-scale and multi-disciplinary area of research interest. As a trigger, 
radiation-induced crucial DNA damage represented by double-strand breaks (DSBs) can be a cause of reproductive cell death $[1,2]$. However, because of the experimental requirements, it is difficult to directly observe and quantify the details of such microscopic lesions. Because of this difficulty, indirect measurements of features related to initial DNA damage, such as physical disconnection of DNA-fiber [3,4], chromosomal aberrations [5], and phosphorylated H2AX as a marker for DSBs [6-8], have been attempted to further investigate radiation-induced DNA damage. Thus, track-structure Monte Carlo (MC) simulations have an important role in investigating DNA damage induced after radiation irradiation [9-11]. In the past decades, many MC codes have achieved successful outcomes for quantitative investigation of radiation-induced initial DNA damage within cellular domains and sub-cellular biological components[12-25].

In a previous study [26], Geant4-DNA simulations [28-31], which are an extension of Geant4 [32-34] for low-energy particle transport, including the simulation of water radiolysis and geometries of biological targets, successfully estimated not only initial DNA damage, but also the fraction of surviving V79 cells by using a two-lesion kinetics (TLK) model [35] that bridges the gap between initial DNA damage and reproductive cell death. Although V79 is one of the most important cell lines in radiation biology research field, it is demanding to extend the application to other cell lines used for evaluation of radiation treatment effects. In general treatment systems, clinically delivered doses to a target tumor are calculated from the so-called "biological dose" which is the absorbed physical dose multiplied by the relative biological effectiveness (RBE) representing the cell-killing effectiveness of the irradiation [36,37]. The RBE tends to be evaluated as the ratio of the cell survival of the reference cell line to the cell survival of cells irradiated with gamma rays [38]. A type of human cancer cell HSGc-C5, is widely used for evaluating the RBE for proton/carbon treatment planning [39-44]. For this reason, we investigated extending the Geant4-DNA application with a TLK model for performance evaluation for DNA damage repair in the HSGc-C5 cell line.

\section{Materials and Methods}

This study comprised two parts: The first part is an experimental study (illustrated in Section 2.1) to measure cell surviving fractions (SFs) and DNA-rejoining kinetics as the reference data for the optimization of the TLK model parameters of HSGc-C5. The second part describes the optimization of the TLK model parameters to evaluate the repair performance of HSGc-C5 (illustrated in Section 2.2). As inputs of the TLK models, the initial DNA damage yields and their details were estimated by performing Geant4-DNA radiobiological simulations.

\subsection{Experimental condition}

\subsubsection{Irradiation condition}

All experiments were performed in the cyclotron facility at the National Institute of Radiological Science (NIRS, QST, Japan). The protons were delivered in the experimental beamline (C8) from the cyclotron (NIRS-930; Thomson CSF, France). A Wobbler method was used to widely spread field of the primary protons [45] to deliver a uniform beam field. The energy of the protons was $70 \mathrm{MeV}$, and the collimated field size was approximately $8 \mathrm{~cm} \times 8 \mathrm{~cm}$ at the isocenter plane. The primary energy of the protons upon cell entrance was changed between binary choice by inserting a $32 \mathrm{~mm}$ thick poly methyl methacrylate (PMMA) block (just before the Bragg peak of a $70 \mathrm{MeV}$ proton in the PMMA block as shown in Figure 2).

\section{Dosimetry}

The beam-monitor count (count/Gy) and the beam-count rate (count/second) were calibrated by using a Markus ion chamber (PTW 23343; PTW, Germany) as shown in panel (A) of Figure 1, and the calibration was repeated for both conditions, with and without the 32-mm thick PMMA block. In the biological assay, the cultured cells were 
Experimental Setup (A)

(B)

(C)
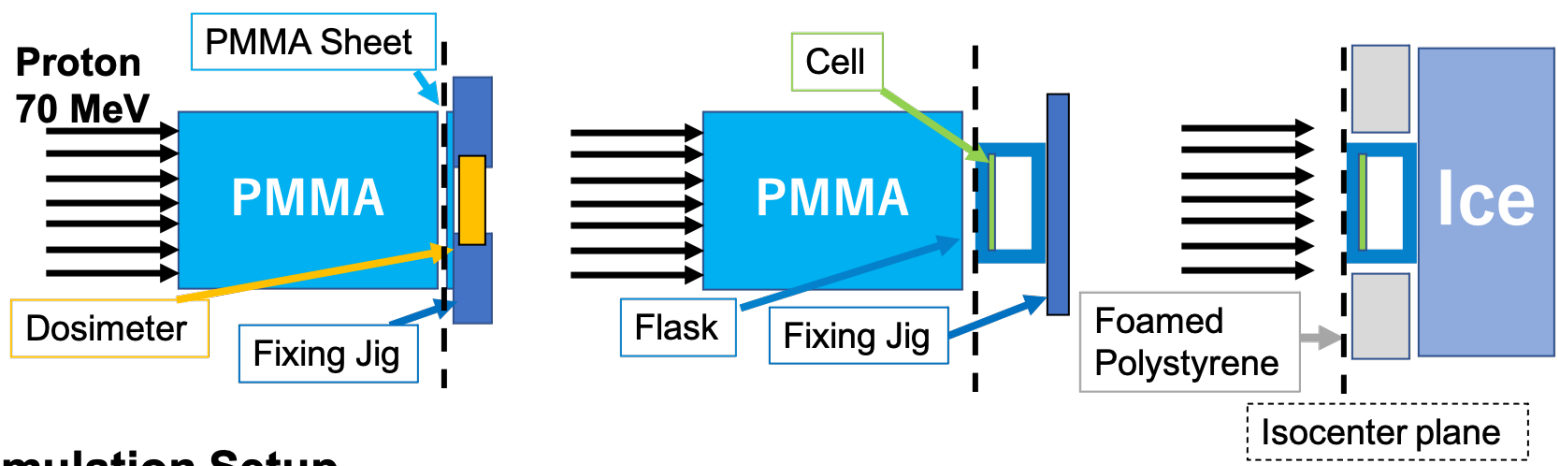

\section{Simulation Setup}

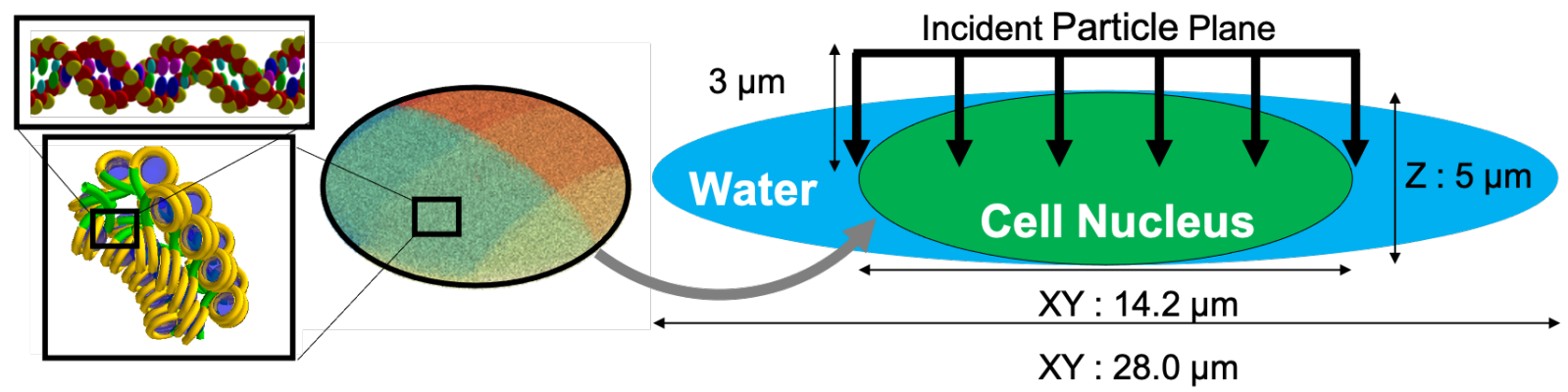

Figure 1. Top (A): Geometrical experimental setup for dosimetry. Top (B): Geometrical experimental setup for colony assay. Top (C): Geometrical experimental setup for the fraction of activity released (FAR) assay. Bottom: Geometrical simulation setup of the irradiated cells and sub-components.

plated on the back side of the upstream window of plastic cell-culture flasks (Falcon 353107/353108; Corning Inc., US), as shown in panels (B) and (C) of Figure 1. Therefore, the water-equivalent thicknesses of the chamber window and of the flask window were different, so a $0.41 \mathrm{~mm}$ thick PMMA sheet was placed in front of the chamber to minimize the thickness difference.

In the simulation study that followed, the energy spectra of protons at the cell entrance level were estimated for the biological simulations. For this purpose, the material properties of PMMA (mass density and mean ionization potential, the so-called I-value) need to be estimated. Thus, we have also measured the percent depth dose (PDD) for $70 \mathrm{MeV}$ protons in PMMA by changing the PMMA thickness $\leq 35 \mathrm{~mm}$ as the reference data for estimation of the material properties with the dosimetry setup.

\section{Colony assay}

Panel (B) of Figure 1 illustrates the schematic experimental setup for the colony assay. A T-25 plastic cell-culture flask was used to place the cells in the irradiation field. A plastic fixing jig was used downstream to fix the flask on the beam line. The target cells were plated on the back side of the window located at the isocenter. To measure cell survival for two different radiation qualities, the irradiation experiments were performed with and without a $32 \mathrm{~mm}$ PMMA block. The delivered dose was chosen as 1, 2, 3, 5, or 7 Gy for the irradiation condition without a PMMA block, and as 1, 2, 3, 4, or 6 Gy for the irradiation condition with a PMMA block. The dose rate was approximately $1 \mathrm{~Gy} / \mathrm{min}$ for all conditions. The irradiation experiments for each condition were repeated three times on the same day, but the experiments with and without a 32 mm PMMA block were performed on different days. 
Gel electrophoresis assay

Panel (C) of Figure 1 illustrates the schematic experimental setup for electrophoresis assay. The target cells are confined in a T-12.5 flask placed at the isocenter. To inhibit DNA rejoining during irradiation and prevent the need to taxi them to the biological work room the flask was cooled on ice. Pre-cooling on ice was also performed from 5 minutes before of the irradiation. To the cell sample, $200 \mathrm{~Gy}$ was delivered at a dose rate of approximately $100 \mathrm{~Gy} / \mathrm{min}$.

\subsubsection{Cell culture and biological processing before irradiation}

We selected a cell line that is a typical benchmark cell in radiation therapy. We selected the HSGc-C5 cell line (No. JCRB1070), distributed by the Japanese Collection of Research Bioresources (JCRB) Cell Bank (National Institutes of Biomedical Innovation, Health, and Nutrition, Japan). The cell line was stored at the NIRS as frozen stocks after culturing in a $5 \% \mathrm{CO}_{2}$ incubator at $37^{\circ} \mathrm{C}$ within Eagle's minimum essential medium containing $60 \mathrm{mg} / \mathrm{L}$ kanamycin, supplemented with 10\% fetal bovine serum (EquitechBio Inc., US). As the first step, approximately 10 days before the irradiation, the frozen stocked cells were unfrozen by placing them in warm water at $37^{\circ} \mathrm{C}$, then inoculated and sub-cultured into T-75 flasks (Falcon 353135; Corning Inc., US). Two days before the irradiation, the sub-cultured cells were trypsinized (2.5\% Trypsin, Thermo Fisher Scientific, US) and inoculated into the T-25 (for the colony assay, Falcon 353018; Corning Inc., US) or T-12.5 (for the FAR assay, Falcon 353107; Corning Inc., US) flask at a density of $3.24 \times 10^{4}$ cells per $\mathrm{cm}^{2}$ as a confluent condition.

\subsubsection{Cell survival measurement}

Within 40 minutes after irradiation, the irradiated cells were taxied to the work room, rinsed with phosphate-buffered saline (PBS), and trypsinized. The experimental procedure performed in this study was described previously in reports by Suzuki $[46,47]$. Then, the trypsinized cells were plated onto plastic culture dishes (Falcon 353002; Corning Inc., US). After the cells are incubated in the $\mathrm{CO}^{2}$ incubator at $37^{\circ} \mathrm{C}$ for approximately 14 days, the colonies were fixed and stained with $20 \%$ methanol containing $0.2 \%$ crystal violet. Any colony consisting of more than $\geq 50$ cells was scored as a surviving clone. The SF was calculated according to the following equation:

$$
\mathrm{SF}=\frac{N_{\mathrm{irr}}^{\mathrm{col}} / N_{\mathrm{irr}}^{\mathrm{plat}}}{N_{\text {nonirr }}^{\text {col }} / N_{\text {nonirr }}^{\text {plat }}},
$$

where $N_{\text {irr }}^{\text {col }}$ is the number of irradiated cells that create a colony, $N_{\text {irr }}^{\text {plat }}$ is the number of cells plated onto the dish after irradiation, $N_{\text {nonirr }}^{\text {col }}$ is the number non-irradiated cells (usually called as control cells) that create a colony, and $N_{\text {nonirr }}^{\text {plat }}$ is the number of control cells plated onto the dish.

\subsubsection{DNA-rejoining kinetics measurement}

The cell sample was kept at $4{ }^{\circ} \mathrm{C}$ during exposures. As for the colony assay, the irradiated cell samples were taxied to the work room within 40 minutes after irradiation but kept on ice to maintain a cold temperature. The experimental procedure performed in this study was described previously in a report by Hirayama [48]. The cells were lysed directly or kept in the incubator to allow DNA rejoining under the aerobic conditions with $5 \% \mathrm{CO}_{2}$ at $37{ }^{\circ} \mathrm{C}$. The cells were washed twice with cold PBS, rinsed with cold $0.05 \%$ trypsin-EDTA, and kept on ice for 20 minutes. The cells were resuspended in cold PBS and embedded in 1\% SeaPlaque GTG agarose gels (50111; Cambrex, US) plugs at a density of approximately $1 \times 10^{5}$ cells $/ \mathrm{ml}\left(1 \times 10^{4}\right.$ cells $\left./ \mathrm{plug}\right)$. All steps were performed on ice to minimize DNA rejoining. The cells in the agarose plugs were incubated in a lysis solution (R\&D Systems, US) containing $0.5 \mathrm{mg} / \mathrm{ml}$ proteinase K (Sigma-Aldrich, 
US) for 1 hour to guarantee the diffusion of chemicals into the agarose. Cell lysis was performed at $50{ }^{\circ} \mathrm{C}$ for 24 hours. The plugs were equilibrated at $\mathrm{pH} 8$ in TE buffer (Sigma-Aldrich; US) for 1 hour at room temperature and used for electrophoresis. The plugs were loaded onto $0.6 \%$ SeaKem Gold agarose gels (50152; Lonza, Switzerland) and subjected to electrophoresis at a field strength of $0.6 \mathrm{~V} / \mathrm{cm}$ in $0.5 \times$ Tris-borate EDTA (TBE) buffer (GeneMax,Taiwan) for 36 hours. The gel was stained for $\geq 3$ hours with ethidium bromide $(2 \mu \mathrm{g} / \mathrm{ml})$ and maintained overnight at room temperature in distilled water. The fluorescence intensities were measured with a UV transilluminator (Mupid-Scope WD; Mupid, Japan) and a digital camera (IXY 220F; Canon, Japan) with an orange filter, which was connected to a computer with image analysis software (1D Image Analysis Software; Kodak, Japan).

The fluorescence intensities of DNA that was retained in the plug and released from the plug were measured by using the image analysis software. The fluorescence intensities for released DNA were proportional to the total amount of DNA fragments, which were separated by physical disconnection of the DNA fiber. The FAR calculation equation was as follows:

$$
\operatorname{FAR}(t)=\frac{I_{\text {out }}(t)}{\left(I_{\text {in }}(t)+I_{\text {out }}(t)\right)}
$$

where, $t$ is the time after irradiation, $I_{\text {in }}(t)$ is the fluorescence intensity of DNA retained in the plug, and $I_{\text {out }}(t)$ is the fluorescence intensity of DNA released from the plug. Then, the relative FAR referenced to FAR ( 0 minutes) as a function of time was calculated.

\subsection{Simulation conditions and model calculation}

\subsubsection{Calculation of the incident proton energy spectra at cell entrance}

To estimate the initial DNA damage by means of Geant4-DNA, the energy of the incident protons at cell entrance was determined using Geant4 (since Geant4-DNA does not support particle transport in PMMA, and the spatial resolution of Geant4 is sufficient to simulate particle transport in millimeter scale volume). In this study, the energy of the incident protons was downscaled by filttering through a $32 \mathrm{~mm}$ PMMA block. Hence, the incident energy of protons was not mono-energetic but was multi-energetic due to the energy loss and straggling in the PMMA block. In addition, even when the PMMA block was not placed upstream, the proton energy spectrum was broadened when the protons passed through the window of the plastic flask. MC simulation is a useful tool for estimating the energy spectra at the cell entrance if the properties of the materials, such as mass density, atomic/molecular composition, and mean ionization potential (so-called I-value), are known when simulations are performed by using the condensed-history approach.

However, in general, it is hard to know the precise material properties of organic materials, such as PMMA. In this study, we have adjusted the material properties, in particular, the I-value, in a way that was consistent with the measured PDD in the PMMA block, by comparing with the Geant 4 simulations. PMMA is a composite material consisting of five carbon atoms, eight hydrogen atoms, and two oxygen atoms. The mass density was selected as the typical PMMA density of $1.190 \mathrm{~g} / \mathrm{cm}^{3}$. The absorbed dose in the sensitive volume of an advanced Markus chamber was simulated for a PMMA block and for a PMMA sheet, with PMMA block thicknesses $\leq 35 \mathrm{~mm}$ to adjust the I-value. For particle transport, QGSP_BIC_EMY is chosen as the condensed-history particle transport model, which is the recommended particle transport model for protons in the clinical energy range [49,50]. During the simulations with the adjusted PMMA properties, the energy of protons at the entrance to the sensitive volume of the advanced Markus chamber is measured. 


\subsubsection{Initial DNA damage}

Simulation using Geant4-DNA

To estimate initial DNA damage, the same simulation configuration as in the previous study was used [23]. The simulation settings are detailed in our previous papers, thus, only brief details are described in this section. As in the previous studies $[22,23]$, a geometrical model of cell that imitates a normal human fibroblast cell was used. The cell geometry is presented in the bottom panel of Figure 1. A cell nucleus $(14.2 \mu \mathrm{m} \times 14.2 \mu \mathrm{m} \times 5.0 \mu \mathrm{m})$ was placed at the center of a water absorber modelling the cytoplasm $(28.0 \mu \mathrm{m} \times 28.0 \mu \mathrm{m} \times 5.0 \mu \mathrm{m})$. In the cell nucleus, a sub-biological component was assembled (the total number of base pairs (bps) was approximately $6.4 \mathrm{Gbp}$ ). A double-helix DNA fiber consists of spherical phosphate/deoxyribose molecules with two ellipsoidal nucleotide bases (the combination of the pair was chosen randomly) as a backbone $[51,52]$ constructed by forming a fractal shape chromatin fibre wrapped by spherical histones $[22,23]$. The incident protons were homogeneously irradiated on the top of the cell nucleus $3.0 \mu \mathrm{m}$ ) away from the center plane to the other side of the cell, as shown by black arrows in the figure. The energy of the protons was randomly chosen from those in the estimated proton energy spectrum for each PMMA thickness illustrated in Section 2.2.1.

For particle transport in cell and reactions with cellular sub-components, the G4EmDNAPhysics_option4 set of physics models, was used [53,54]. The production and reaction schemes of chemical species during radiolysis were simulated with the independent-reaction time (IRT) method $[55,56]$. The direct and indirect DNA damage models were adjusted in the previous study [23]. The adjustment in the proportional probability direct damage model from 0 at $5 \mathrm{eV}$ to 1 at $37.5 \mathrm{eV}$ was selected as originally proposed by PARTRAC [16]. As in the previous study [23], we set 0.405 as the probability of a chemical reaction between a hydroxyl radical and the sugar-phosphate backbone resulting in an indirect damage. The histones placed in the cell model are modeled as perfect scavengers for all radiolytic species.

\section{DNA damage classification}

The initial DNA damage needs to be classified into two components because the TLK model considers two types of repair kinetics. As an assumption, we considered that all simple DSBs are repaired by the fast-repair process, and all complex DSBs are repaired by the slow-repair process. In this study, as in the previous study, a classification scheme originally proposed by Nikjoo et al [14] for DSB damage classification was used. Simple DSBs can be considered to be two-strand breaks (SB) on opposite strands within a short distance (typically within $10 \mathrm{bps}$ ) from each other. We considered two damage types as complex DSBs. $\mathrm{A} \mathrm{DSB}_{+}$requires a DSB and at least one additional SB within $10 \mathrm{bp}$, whereas a $\mathrm{DSB}_{++}$requires two DSBs or more along the chromatin fibre segment. Each damage cluster is defined as different from another if no damage can be found in 100 consecutive bps.

\subsubsection{Evaluation of repair performance}

\section{TLK model}

The TLK model proposed by Stewart [35] represents the kinetic processes of fastand slow-DNA repair as well as the subsequent SF calculated in accordance with their residual lethal DNA damage. The number of lesions induced by radiation increases during the irradiation, then such lesions repaired in the following DNA repair processes over time. Both fast- and slow-repair consider simple rejoining of bp-break ends at the same position through the corresponding repair process, expressed in terms of either single-order repair in which $L(t)$ represents the yield of lesion is proportional to time $t$ or as multiple-lesion repairs (second-order repair) in response to a complex aberration, possibly by incorrect rejoining of the break ends with two different lesions (binary mis-repair)) expressed as $L(t) L(t)$. 
In this study, the six parameters TLK model was applied by using the same approach as used in the previous studies with V79 cells $[26,27]$ as well as the original study by Stewart [35]. The model parameters can be categorized into two types: 1) repair probability coefficients, which represent the fraction of rejoined lesions in a unit of time $(\lambda$ or $\eta$ ), and 2) parameters for lethality which represent the probability of the residual lesion leading to cell death $(\beta$ or $\gamma)$. With these considerations, the six parameters TLK model can be written as follows,

$$
\begin{aligned}
& \frac{d L_{1}(t)}{d t}=D(t) Y \Sigma_{1}-\lambda_{1} L_{1}(t)-\eta L_{1}\left[L_{1}(t)+L_{2}(t)\right], \\
& \frac{d L_{2}(t)}{d t}=D(t) Y \Sigma_{2}-\lambda_{2} L_{2}(t)-\eta L_{2}\left[L_{1}(t)+L_{2}(t)\right],
\end{aligned}
$$

and

$$
\frac{d L_{f}(t)}{d t}=\beta_{1} \lambda_{1} L_{1}(t)+\beta_{2} \lambda_{2} L_{2}(t)+\gamma \eta\left[L_{1}(t)+L_{2}(t)\right]^{2} .
$$

Here $L_{1}(t)$ is the number of lesions in fast-repair per cell at a time $t$ from the start of irradiation, similarly, $L_{2}(t)$ is the number of lesion in slow-repair per cell at $t$, and $L_{f}(t)$ is the number of lethal lesions leading to cell death at $t . D(t) Y \Sigma_{1}$ and $D(t) Y \Sigma_{2}$ are the lesion production terms for fast- and slow- lesions, respectively, which are proportional to the dose rate $D(t)$ multiplied by instantaneous lesions in a unit dose rate per unit number of bps $\Sigma \mathrm{Gy}^{-1} \mathrm{Gbp}^{-1}$ and number of bps in a cell $Y \mathrm{Gbp}$. In this study, we assumed that all simple DSBs underwent the fast-repair process, and that all complex DSBs underwent that slow-repair process. Thus, the instantaneous lesions are defined as $\Sigma_{1}=N_{D S B}$ and $\Sigma_{2}=N_{D S B+}+2 N_{D S B++}$, where the $\mathrm{N}_{D S B}, \mathrm{~N}_{D S B_{+}}$and $\mathrm{N}_{D S B_{++}}$are the number of simple DSBs, $\mathrm{DSB}_{+}$, and $\mathrm{DSB}_{++}$, respectively, as in Nikjoo's definition. $\lambda_{1}, \lambda_{2}$ and $\eta$ are the probabilities of rejoined lesions $\left(\mathrm{h}^{-1}\right)$ by the fast-, slow-, and binaryrejoining processes, respectively. Similarly, $\beta_{1}, \beta_{2}$, and $\gamma$ represent the probabilities of the residual lesions leading to cell death for each rejoining process. In this study, as in the previous studies [26,35], $\beta_{1}$ was forced to 0 , since in general, simple-DSBs do not have much of an effect on cell survival $\geq 2$ weeks after irradiation. If the first-order repair is not saturated, the half-life time $\tau$ of the rejoining can be calculated by $\tau=\ln 2 / \lambda$.

Finally, these yields were numerically integrated to calculate SF

$$
\mathrm{SF}(t)=\ln \left(-L_{f}(t)\right)
$$

The differential equation has been solved numerically by means of the 4th order RungeKutta method in the boost/numerical $\mathrm{C}++$ library. The SF is calculated at $t=336$ hours since the number of colonies is counted after 14 days from the irradiation in the experimental assay. Additionally, $D(t)$ is set to $60 \mathrm{~Gy} / \mathrm{h}$ until the target dose is delivered. The time step of the integration is set to $1 \times 10^{-4}$ hour.

\section{Random-breakage model}

FAR is a method for quantitative investigation of the number of fragments separated by physical disconnection of DNA fiber, such as DSBs. According to the randombreakage model [57-59], the equation can convert the number of the unrejoined DSBs $\left(\left(L_{1}(t)+L_{2}(t)\right) / Y\right)$ in the FAR is given by the following equation:

$$
\operatorname{FAR}(t)=F_{m a x}\left\{1-\left[1+K\left(L_{1}(t)+L_{2}(t)\right) / Y\left(1-\frac{K}{M_{0}}\right)\right] \exp ^{-K\left(L_{1}(t)+L_{2}(t)\right) / Y}\right\}
$$

where $F_{\text {max }}$ is the maximum fraction of the DNA that can enter the gel plug, $M_{0}$ is the average DNA length in a chromosome, and $K$ is the detection limit length (DNA fragments shorter than $K$ do not move out of the gel wall). In this study, $F_{\text {max }}$ was set to $1, M_{0} \sim 139 \mathrm{Mbp}$ (6.4 Gbp/46 chromosomes) and $K=1 \mathrm{Mbp}$ is the limit of the FAR assay (measured with the fiducial DNA marker). To match the experimental definition, 
to calculate the relative FAR, the FAR values were scaled by applying $\operatorname{FAR}\left(t_{0}\right)$, where $t_{0}$ is the time when irradiation stopped.

\section{Parameter optimization}

To evaluate the repair performances represented as $\lambda_{1}, \lambda_{2}, \eta, \beta_{1}, \beta_{2}$ and $\gamma$, these model parameters in Eqs.(3)-(5) were optimized for HSGc-C5, in a way that was consistent with the experimentally measured SF and relative FAR. To optimize the parameters, we performed Ceres Solver [60], which is an open-source C++ library based on the nonlinear least-squares method for solving optimization problems. SPARSE_NORMAL_CHOLESKY was selected as the algorithm of the solver. The residual cost for each data point was calculated as $V_{\text {calc }}-V_{\text {exp }}$, where $V_{\text {calc }}$ was calculated as the value with simulated DSBs and $V_{\text {exp }}$ was calculated as the value of experimental data with the same weight all configurations of both the SF and relative FAR.

\section{Results}

\subsection{Incident energy spectra at cell entrance}

To estimate the energy spectra of the incident protons at cell entrance in the irradiation experiments for the colony assay and FAR assay, the I-value of the PMMA used in the experiments was estimated. After adjustment to gain agreement with the measured PDD, we selected $65 \mathrm{eV}$ as the I-value. Figure 2 shows the PDD of $70 \mathrm{MeV}$ protons in PMMA. The Bragg peak of $70 \mathrm{MeV}$ protons occurred between PMMA from $32 \mathrm{~mm}$ to $33 \mathrm{~mm}$ thick. Thus, in this study, we selected a PMMA thickness of $32 \mathrm{~mm}$ to downscale the energy of the incident protons.

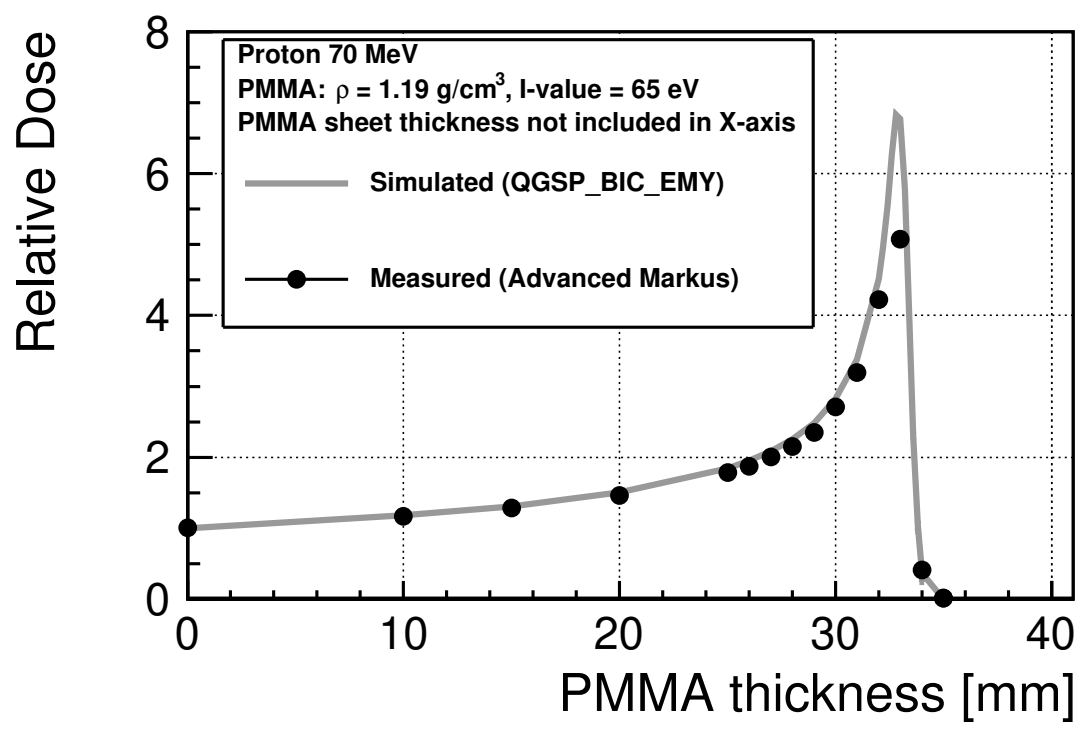

Figure 2. Percent depth dose (PDD) of $70 \mathrm{MeV}$ protons in PMMA.

Figure 3 shows the energy spectra of the incident protons at the entrance (the thickness of PMMA sheet is not considered), scaled to the maximum of the number of protons at a $0 \mathrm{~mm}$ PMMA thickness. The energy is downscaled with increasing PMMA thickness, and the width of a spectrum broadens due to the energy losses and energy straggling while passing through the PMMA. Even when the PMMA block is not inserted, the protons will lose their energy when passing through the PMMA sheet and the window of the advanced Markus chamber. The average proton energies were approximately $68.5,18.7$, and $10.8 \mathrm{MeV}$, and the standard deviations of the spectra were $0.5,1.7$, and $2.1 \mathrm{MeV}$ at PMMA thicknesses of 0,30 , and $32 \mathrm{~mm}$, respectively. The 
corresponding unrestricted linear energy transfer $\left(\mathrm{LET}_{\infty}\right)$ values were $0.05,0.60$ and $0.96 \mathrm{keV} / \mu \mathrm{m}[61]$, respectively.

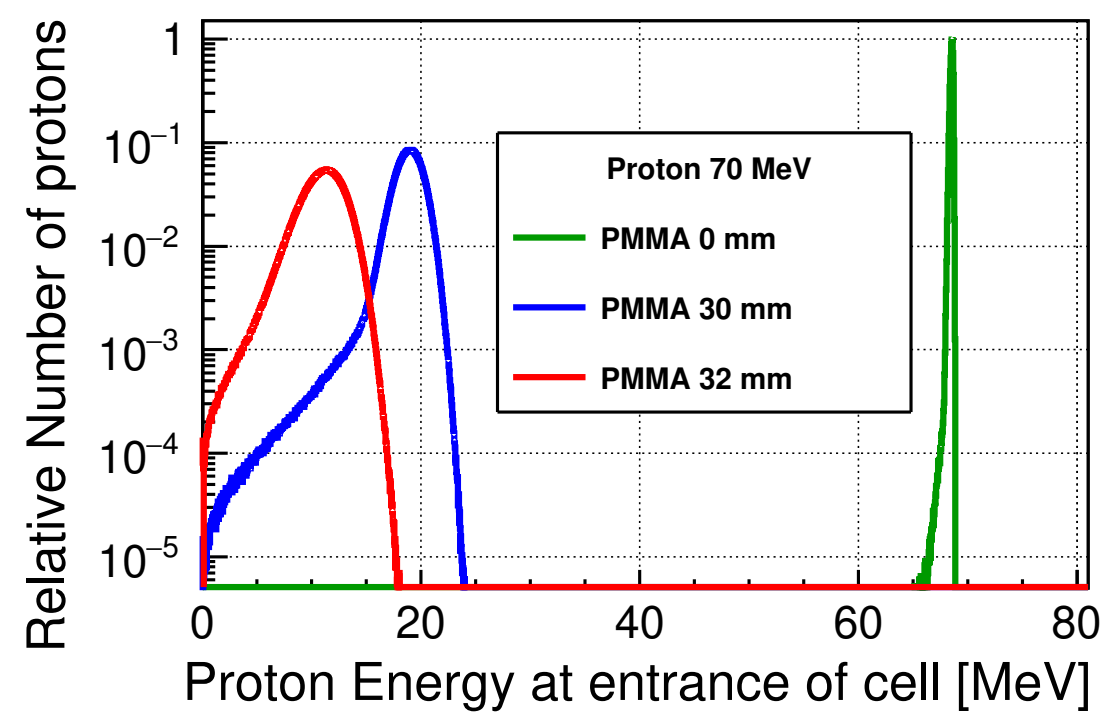

Figure 3. Scaled energy spectra of the protons at the cell entrance, downscaled by a PMMA block and a PMMA sheet. The thickness of the PMMA sheet is not considered in the figure legend.

\subsection{Initial DNA damage}

The simulated initial number of DSBs for each damage type is shown in Figure 4 . For all damage types, the numbers of DSBs were slightly larger with a $32 \mathrm{~mm}$ PMMA block than the numbers without a PMMA block. The average numbers of DSBs were 4.09 and $0.72 \mathrm{~Gy}^{-1} \mathrm{Gbp}^{-1}$ for simple-DSB and complex-DSB without a PMMA block, respectively. With a PMMA block, the average numbers of DSBs were 4.69 and 1.04 $\mathrm{Gy}^{-1} \mathrm{Gbp}^{-1}$, respectively.

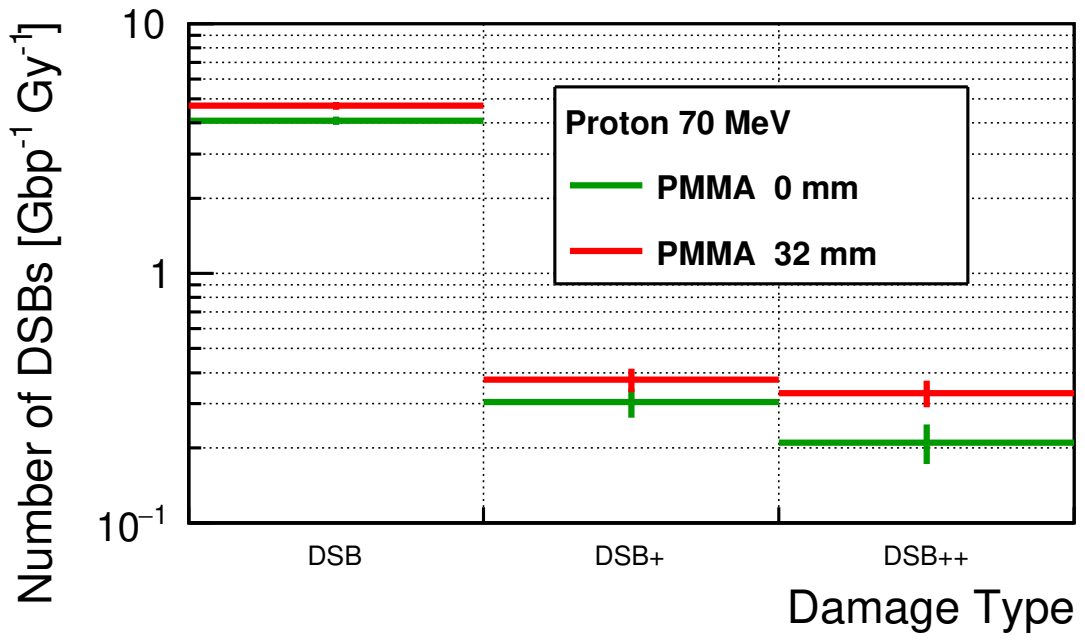

Figure 4. The simulated number of DSBs for each damage type.

\subsection{Optimized repair performance}

As shown in Figure 5, the model parameters are reasonably optimized to reproduce both SF and relative FAR. In other words, using the optimized parameters, Geant4-DNA 
simulations are now able to predict cell survival and DNA-rejoining kinetics of HSGc-C5 via a model calculation with a TLK model.
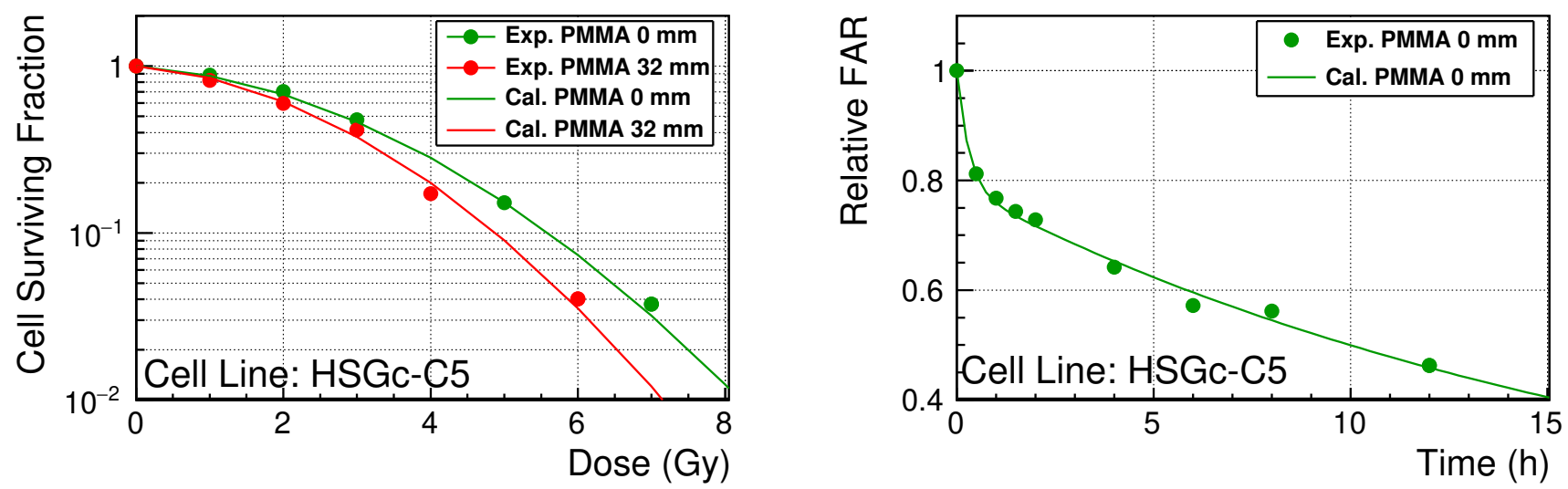

Figure 5. Left: SF of HSGc-C5 as a function of delivered dose. Right: Relative FAR of HSGc-C5 as a function of time after irradiation. The curve is calculated with the optimized TLK model parameters from the simulated initial DNA damage.

The optimized parameters are presented in Table 1. Through the fast-repair process, the probability of the repair was approximately $3.3 \mathrm{~h}^{-1}$ (the half-life time is approximately 12.7 minutes). Through the slow-repair process, the probability of the repair was approximately $0.01 \mathrm{~h}^{-1}$ (the half-life time is approximately 65.1 hours). The probability of the binary repair was significantly small $\left(4.3912 \times 10^{-6} \mathrm{~h}^{-1}\right)$ relative to that of single rejoining. In contrast, the lethality of binary-repair was very high (probability of repair leading cell death, possibly mis-repair, in the binary-repair process $\sim 45 \%$ ). Compared with the lethality of binary repair, the lethality of residual complex DSBs was relatively small $(\sim 3 \%)$.

Table 1: Optimized repair parameters [HSGc-C5]

\begin{tabular}{l|l|l|l|l|l}
\hline$\lambda_{1}\left(\mathrm{~h}^{-1}\right)$ & $\lambda_{2}\left(\mathrm{~h}^{-1}\right)$ & $\eta\left(\mathrm{h}^{-1}\right)$ & $\beta_{1}$ & $\beta_{2}$ & $\gamma$ \\
\hline 3.2763 & $1.0651 \times 10^{-2}$ & $4.3912 \times 10^{-6}$ & 0. & $2.7773 \times 10^{-2}$ & 0.4500 \\
\hline
\end{tabular}

\section{Discussion}

As shown in Figure 4, the differences among the number of DSBs for each damage type appear to be small but this is not surprising, when considering LET value at this energy $\left(\mathrm{LET}_{\infty}=0.96 \mathrm{keV} / \mu \mathrm{m}\right.$ at $10.8 \mathrm{MeV}$ [61]). However, considering only complex DSBs, the number of DSBs was increased $43 \%$ when a PMMA block was inserted. When a $32 \mathrm{~mm}$ PMMA block was inserted and the PMMA thickness is close to the Bragg peak position, the average energy of the protons was approximately $10.8 \mathrm{MeV}$ (both the CSDA range and projected range of $10.8 \mathrm{MeV}$ protons in PMMA were approximately $0.52 \mathrm{~mm}$ [61]). This fact also means that it is hard to downscale the energy of protons more using PMMA blocks at $1 \mathrm{~mm}$ step. Hence, to perform the experiments for higherLET protons, we need to perform the experiments with thinner PMMA blocks, otherwise we need to use low energy proton beam facilities.

In general, cells rely on two highly regulated DSB repair pathways: the nonhomologous end-joining (NHEJ) pathway and homologous recombination (HR) pathway. Our results indicate that the HSG cell is also relies on the NHEJ and HR for DNA damagerepair processes. This was more evident when we compared the speed of the rejoining with the measured repair speed. According to the experimental measurements, NHEJ processes can be completed in approximately 0.5 hour, whereas HR is much slower and takes dozens of hours to complete [62]. In the case of HSGc-C5, the fast-repair process 
was very fast. This fact indicates, even when we consider the lethality of residual simple DSBs, the number of residual lethal lesion can be very small 2 weeks after the irradiation. Hence, the simple DSBs should not have much of an effect on the SF calculations, as we ignored in this study. This insensitivity of the fast-repair component is briefly discussed in the appendix A. HR is regarded as one of the most accurate repair processes. According to the estimated lethality, only $3 \%$ of repaired lesions through the HR process lead to reproductive cell death of HSGc-C5 cells. This finding indicates that, assuming of that all mis-repaired lesions lead to cell death, HR can repair $~ 97 \%$ of complex DSBs. However, repairing binary lesions is rather difficult. Even if the cell repaired a binary lesions, $45 \%$ of rejoined DNA can be mis-repaired and leads to cell death.

\section{Conclusions}

To evaluate the repair performance of $\mathrm{HSGc}-\mathrm{C} 5$ cell against radiation induced DNA damage, the Geant4-DNA application was extended by using newly measured experimental data acquired in this study. Concerning fast- and slow-DNA rejoining, the TLK model parameters were adequately optimized (the repair speeds of each process were reasonably close to the DNA-rejoining speed of the NHEJ and HR processes). The lethality rates of the DNA damage induced by complex DSBs and binary-repair were approximately $3 \%$ and $45 \%$, respectively. Using the optimized repair parameters, the Geant4-DNA simulation was able to predict the SF and the DNA-repair kinetics.

Author Contributions: Conceptualization, D.S. and S.I.; methodology, D.S.; validation, D.S., M.S. and R.H.; formal analysis, D.S.; investigation, S.I. and T.I.; computing resources, T.I.; data curation, D.S, M.S., R.H.,Y.A.,M.M,S.S; writing-original draft preparation, D.S.; writing-review and editing, I.K.,D.E.,S.G,S.I and T.I.; visualization, D.S.; supervision, T.I.; project administration, D.S and S.I.; funding acquisition, D.S. and T.I. All authors have read and agreed to the published version of the manuscript.

Funding: This work was funded by JSPS KAKENHI, Japan, grant number JP20K16840. This work was partially supported by QST President's Strategic Grant (Creative Research).

Conflicts of Interest: The authors declare no conflict of interest.

\section{Abbreviations}

The following abbreviations are used in this manuscript:

$\begin{array}{ll}\text { bp } & \text { base pair } \\ \text { DSB } & \text { double-strand break } \\ \text { MC } & \text { Monte Carlo } \\ \text { TLK } & \text { two-lesion kinetics } \\ \text { RBE } & \text { relative biological effectiveness } \\ \text { PMMA } & \text { poly methyl methacrylate } \\ \text { PDD } & \text { percent depth dose } \\ \text { SF } & \text { surviving fraction } \\ \text { FAR } & \text { fraction of activity released } \\ \text { LET } & \text { linear energy transfer } \\ \text { NHEJ } & \text { non-homologous end-joining } \\ \text { HR } & \text { homologous recombination }\end{array}$

\section{Appendix A. Limitation of the application}

The TLK model considers only two major repair pathways, however, for human cells, the alternative-NHEJ and single-strand annealing can be important pathways [63]. If the number of dominant repair pathways is more than two, the TLK model cannot be used to evaluate repair performance and cell survival. As an example, the study was repeated for a type of normal human skin fibroblast cells, named NB1RGB (No. RCB0222), distributed by the RIKEN BioResource Center Cell Bank (RIKEN, Japan). The TLK model could not adequately describe the DNA-rejoining kinetics of NB1RGB, as shown in the 
right panel of the Figure 1, although the model can be optimized for SF, as shown in the left panel of the Figure 1.
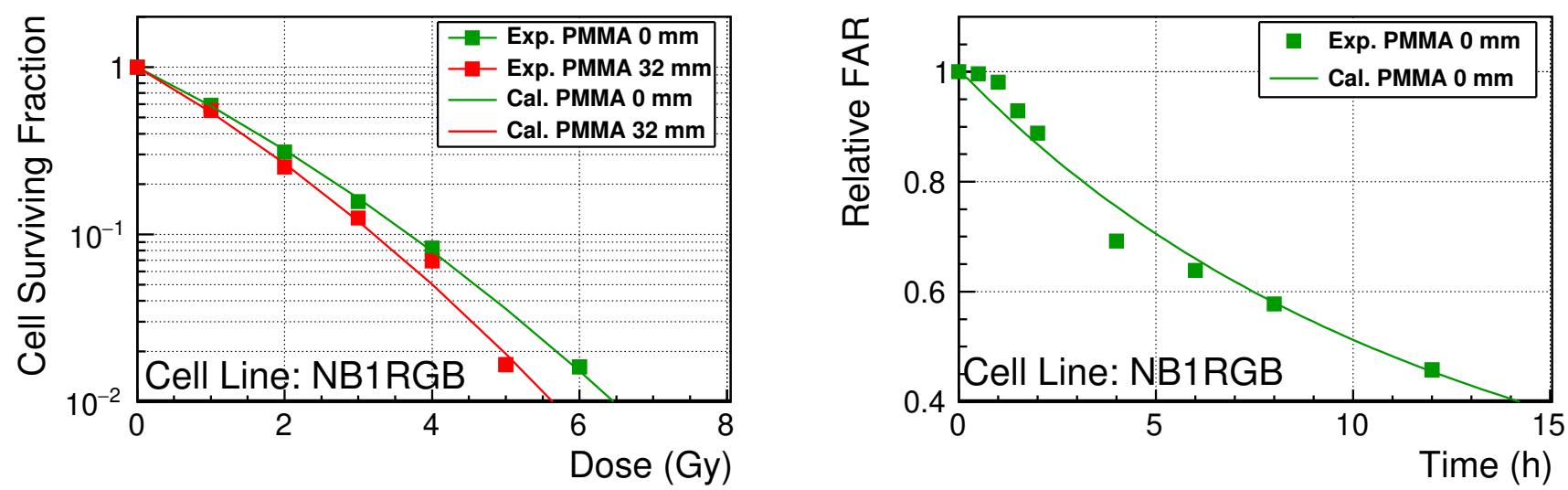

Figure 1. Left: SF of NB1RGB as a function of the delivered dose. Right: Relative FAR of NB1RGB as a function of time after irradiation. The calculated curve is calculated byusing the optimized TLK parameters from the simulated initial DNA damage.

Table 2: Optimized repair parameters [NB1RGB]

\begin{tabular}{l|l|l|l|l|l}
\hline$\lambda_{1}\left(\mathrm{~h}^{-1}\right)$ & $\lambda_{2}\left(\mathrm{~h}^{-1}\right)$ & $\eta\left(\mathrm{h}^{-1}\right)$ & $\beta_{1}$ & $\beta_{2}$ & $\gamma$ \\
\hline 33062.9 & $1.2540 \times 10^{-2}$ & $7.5701 \times 10^{-6}$ & 0. & $1.9552 \times 10^{-2}$ & 0.1914 \\
\hline
\end{tabular}

It is possible for the optimized parameters to have unsubstantial effects. In the case of NB1RGB cells, the optimized TLK model predicted that $3 \times 10^{6} \%$ simple-DSBs can be repaired in 1 hour, even if the value is not realistic. Comparing with the results of HSGc-C5, the order of magnitude of $\lambda_{2}, \eta, \beta_{1}, \beta_{2}$, and $\gamma$ were the same even though $\lambda_{1}$ is 1000 times larger than $\lambda_{1}$ of HSGc-C5 cells. The SF is calculated from the residual lethal lesions at 2 weeks after the irradiation and is not sensitive to the fast-DNA-repair process. To overcome this limitation of the TLK model, further study considering minor repair pathways, as in the approach used in the study by Belov [64], might be required.

\section{References}

1. Baskar, R. Lee, K. A. Yeo, R. and Yeoh, K-W.Cancer and Radiation Therapy: Current Advances and Future Directions. Int J Med Sci. 2012, 9, 193-199.

2. Rich, T. Allen, R.L. and Wyllie, A.H. Defying death after DNA damage. Nature 2000, 408, 777-783.

3. Aaij C. and Borst P. The gel electrophoresis of DNA. Biochim. Biophys. Acta (BBA) Nucleic Acids Protein Synth 1972, $269,192-200$.

4. Iliakis, G. E., Cicilioni, O. \& Metzger, L. Measurement of DNA double strand breaks in CHO cells at various stages of the cell cycle using pulse field gel electrophoresis: Calibrations by means of 125I decay. Int. J. Radiat. Biol. 1972, 59, $343-357$.

5. Munne, S. Chromosome abnormalities and their relationship to morphology and development of human embryos. Rep. Biomed Online. 2006, 12, 234-253.

6. Paul, T. T. et al. A critical role for histone H2AX in recruitment of repair factors to nuclear foci after DNA damage. Curr. Biol. 2000, 10, 886-895.

7. Rogakou, E. P. et al. DNA double-stranded breaks induce histone H2AX phosphorylation on serine 139. J. Biol. Chem. 1998, 273, 5858-5868.

8. Rogakou, E. P., Boon, C., Redon, C. \& Bonner, W. M. Megabase chromatin domains involved in DNA double-strand breaks in vivo. J. Cell Biol. 1999, 146, 905-916.

9. Nikjoo, H. et al. Track structure in radiation biology: theory and applications. Int. J. Radiat. Biol. 1998, 73, 355-364.

10. Nikjoo, H., Taleei, R., Liamsuwan, T., Liljequist D., Emfietzoglou, D., Perspectives in radiation biophysics: From radiation track structure simulation to mechanistic models of DNA damage and repair. Rad. Phys. Chem. 2016, 128, 3-10.

11. Nikjoo, H. et al. Radiation track, DNA damage and response-a review. Rep. Prog. Phys. 2016, 79, 116601.

12. Uehara, S., Nikjoo, H. and Goodhead, D. T. Cross-section of water vapour for the Monte Carlo electrons track structure code from $10 \mathrm{eV}$ to the MeV region. Phys. Med. Biol. 1993, 38, 1841-1858. 
13. Nikjoo, H., O'Neill, P., Goodhead, D. T. \& Terrissol, M. Computational modeling of low-energy electron-induced DNA damage by early physical and chemical events. Int. J. Radiat. Biol. 1997, 71, 467-483.

14. Nikjoo, H., O'Neill, P., Wilson, W. E. \& Goodhead, D. T. Computational approach for determining the spectrum of DNA damage induced by ionizing radiation. Rad. Res. 2001, 156, 577-583.

15. Friedland, W. et al. Monte Carlo simulation of the production of short DNA fragments by low-linear energy transfer radiation using higher-order DNA models. Rad. Res. 1998, 150, 170-182.

16. Friedland, W. et al. Simulation of DNA Damage after Proton Irradiation. Rad. Res. 2003, 159, 401-410.

17. Friedland, W., Dingfelder, M., Kundrát, P. and Jacob, P. Track structures, DNA targets and radiation effects in the biophysical Monte Carlo simulation code PARTRAC. Mutat. Res. 2011, 711, 28-40.

18. Friedland, W. et al. Comprehensive track-structure based evaluation of DNA damage by light ions from radiotherapy-relevant energies down to stopping. Sci. Rep. 2017, 7, 45161.

19. Plante, I. and Francis, A. Applications of Monte Carlo Methods in Biology, Medicine and Other Fields of Science. InTech; 2011, Monte-Carlo Simulation of Ionizing Radiation Tracks; 315-356.

20. Plante, I. and Devroye, L. Considerations for the Independent Reaction Times and Step-by-Step Methods for Radiation Chemistry Simulations. Radiation Physics and Chemistry. 2016, 157-172.

21. Meylan, S. et al. Simulation of early DNA damage after the irradiation of a fibroblast cell nucleus using Geant4-DNA. Sci. Rep. 2017, 7, 11923.

22. Sakata, D. et al. Evaluation of early radiation DNA damage in a fractal cell nucleus model using Geant4-DNA. Phys. Med. 2019, 62, 152-157.

23. Sakata, D. et al. Fully integrated Monte Carlo simulation for evaluating radiation induced DNA damage and subsequent repair using Geant4-DNA Sci. Rep. 2020, 10, 20788.

24. Schuemann, J. et al. TOPAS-nBio: An Extension to the TOPAS Simulation Toolkit for Cellular and Sub-cellular Radiobiology. Rad. Res. 2018, 191, 125-138.

25. McNamara, A. L. et al. Geometrical Structures for Radiation Biology Research as Implemented in the TOPAS-nBio Toolkit. Phys. Med. Biol. 2018, 63, 175018.

26. Sakata, D. et al. Evaluation of DNA rejoining and cell survival for V79 cell using Geant4-DNA. The manuscript will be soon submitted to Phys. Med..

27. Tang N. Évaluation, à partir de modélisations nanodosimétriques, de l'influence de la compaction de la chromatine sur les effets radio-induits précoces et extension aux effets tardifs (réparation des dommages à l'ADN et mort cellulaire). ph.D thesis (Written in French) from the University of Bordeaux.

28. Incerti, S. et al. The Geant4-DNA project. Int. J. Model. Simul. Sci. Comput. 2009, 1, 157-178.

29. Incerti, S. et al. Comparison of Geant4 very low energy cross section models with experimental data in water. Med. Phys. 2010, 37, 4692-4708.

30. Bernal, M. A. et al. Track structure modeling in liquid water: A review of the Geant4-DNA very low energy extension of the Geant4 Monte Carlo simulation toolkit. Phys. Med. 2015, 31, 157-178.

31. Incerti, S. et al. Geant4-DNA example applications for track structure simulations in liquid water: a report from the Geant4-DNA Project. Med. Phys. 2018, 45, e722-e739.

32. Agostinelli, S. et al. Geant4 - A Simulation Toolkit. Nucl. Instrum. Meth. A 2003, 506, 250-303.

33. Allison, J. et al. Geant4 developments and applications. IEEE Trans. Nucl. Sci. 2006, 53, 270-278.

34. Allison, J. et al. Recent Developments in Geant4. Nucl. Instrum. Meth. A 2016, 835, 186-225.

35. Stewart, R. D. Two-Lesion Kinetic Model of Double-Strand Break Rejoining and Cell Killing. Rad. Res. 2001, 156, 365-378.

36. Inaniwa, T. et al. Reformulation of a clinical-dose system for carbon-ion radiotherapy treatment planning at the National Institute of Radiological Sciences, Japan. Phys. Med. Biol. 2015, 60, 3271.

37. Inaniwa, T. et al. Treatment planning of intensity modulated composite particle therapy with dose and linear energy transfer optimization. Phys. Med. Biol. 2017, 62, 5180.

38. Scholz, M. and Kraft, G. Track structure and the calculation of biological effects of heavy charged particles Adv. Space Res. 1996, $18,5-14$.

39. Furusawa, Y. et al. Inactivation of Aerobic and Hypoxic Cells from Three Different Cell Lines by Accelerated ${ }^{3} \mathrm{He}-,{ }^{12} \mathrm{C}-$ and ${ }^{20}$ Ne-Ion Beams. Rad. Res. 2000, 154, 485-496.

40. Ando, K. et al. Relative biological effectiveness of the $235 \mathrm{MeV}$ proton beams at the National Cancer Center Hospital East. J Radiat Res 2001, 42, 79-89.

41. Kase, Y. et al. Microdosimetric measurements and estimation of human cell survival for heavy-ion beams. Rad. Res. 2006, 166, $629-638$.

42. Uzawa, A. et al. Comparison of biological effectiveness of carbon-ion beams in Japan and Germany. Int. J. Radiat. Oncol. Biol. Phys. 2009, 73, 1545-1551.

43. Matsuura, T. et al. Apparent absence of a proton beam dose rate effect and possible differences in RBE between Bragg peak and plateau. Med. Phys. 2010, 37, 5376-5381.

44. Okamoto, H. et al. Relation between lineal energy distribution and relative biological effectiveness for photon beams according to the microdosimetric kinetic model. J Radiat Res 2010, 52, 75-81. 
45. Koehler, A. M., Schneider, R. J., and Sisterson, J. M., Range modulators for protons and heavy ions. Nucl. Instrum. Meth. 1975, 131, 437-440.

46. Suzuki, M. et al. Relative biological effectiveness for cell-killing effect on various human cell lines irradiated with heavy-ion medical accelerator in Chiba (HIMAC) carbon-ion beams Int. J. Radiat. Oncol. Biol. Phys. 2000, 48, 241-250.

47. Suzuki, M. et al. Lethal and mutagenic bystander effects in human fibroblast cell cultures subjected to low-energy- carbon ions Int. J. Radiat. Biol. 2019, 96, 179-186.

48. Hirayama, R. et al. Repair kinetics of DNA-DSB induced by X-rays or carbon ions under oxic and hypoxic conditions. J. Radiat. Res. 2005, 46, 325-332.

49. Resch, A.F. et al. Evaluation of electromagnetic and nuclear scattering models in GATE/Geant4 for proton therapy. Med. Phys. 2019, 46, 2444-2456.

50. Lazarakis, P. et al. Investigation of track structure and condensed history physics models for applications in radiation dosimetry on a micro and nano scale in Geant4 Biomed. Phys. Eng. Express 2018, 4, 024001.

51. Lampe, N. et al. Mechanistic DNA damage simulations in Geant4-DNA part 1: A parameter study in a simplified geometry. Phys. Med. 2018, 48, 135-145.

52. Lampe, N. et al. Mechanistic DNA damage simulations in Geant4-DNA part 2: Electron and proton damage in a bacterial cell. Phys. Med. 2018, 48, 146-155.

53. Emfietzoglou, D. Inelastic cross-sections for electron transport in liquid water: a comparison of dielectric models. Radiat. Phys. Chem. 2003, 66, 373-385.

54. Kyriakou, I., Šefl1, M., Nourry, V. and Incerti, S. The impact of new Geant4-DNA cross section models on electron track structure simulations in liquid water. J. Appl. Phys. 2016, 119, 194902.

55. Green,N. J. B., Pilling, M. J., Pimblott, S. M., \& Clifford, P. Stochastic modeling of fast kinetics in a radiation track. J Phys Chem. 1990, 94, 251-258.

56. Karamitros, M. et al. Implementing the Independent Reaction Time method in Geant4 for radiation chemistry simulations. 2020, arXiv:2006.14225.

57. Contopoulou, C. R. Cook, V. E. and Mortimer, R. K. Analysis of DNA double strand breakage and repair using orthogonal field alternation gel electrophoresis. Yeast 1987, 3, 71-76.

58. Cook, V. E. and Mortimer, R. K. A quantitative model of DNA fragments generated by ionizing radiation, and possible experimental applications. Rad. Res. 1991, 125, 102-106.

59. Belli, M. et al. DNA DSB induction and rejoining in V79 cells irradiated with light ions: A constant field gel electrophoresis study. Int. J. Radiat. Biol. 2000, 76, 1095-1104.

60. http://ceres-solver.org

61. Report 90: Key Data for Ionizing-Radiation Dosimetry: Measurement Standards and Applications. J. Int. Com. Rad. Units Meas. 2016, 14, 1 .

62. Zhiyong, M. et al. Comparison of nonhomologous end joining and homologous recombination in human cells. DNA Repair (Amst). 2008, 7, 1765-1771.

63. Hanscom, T. and McVey, M. Regulation of Error-Prone DNA Double-Strand Break Repair and Its Impact on Genome Evolution. Cells 2020, 9, 1657.

64. Belov, O. V. et al. A quantitative model of the major pathways for radiation-induced DNA double-strand break repair. J. Theo. Biol. 2015, 366, 115-130. 